\title{
Vulnerability of Smart Grids with Variable Generation and Consumption: a System of Systems Perspective
}

\author{
Enrico Zio, Senior Member, IEEE, and Giovanni Sansavini
}

\begin{abstract}
This paper looks into the vulnerabilities of the electric power grid and associated communication network, in the face of intermittent power generation and uncertain demand within a complex network framework of analysis of smart grids. The perspective is typical for the system of systems analysis of interdependencies in a critical infrastructure (CI), i.e. the smart grid for electricity distribution. We assess how the integration of the two systems copes with requests to increase power generation due to enhanced power consumption at a load bus. We define adequate measures of vulnerability to identify the most limiting communication time delays. We quantify the probability that a reduction in the functionality of the communication system yields a faulty condition in the electric power grid, and find that a factual indicator to quantify the coupling strength between the two networks is the frequency of load-shedding actions due to excessive communication time delay. We evaluate safety margins with respect to communication specifications, i.e. the data rate of the network, to comply with the safety requirements in the electric power grid. Finally, we find a catastrophic phase transition with respect to this parameter, which affects the safe operation of the CI.
\end{abstract}

Index Terms-Complex Networks, System of Systems, Critical Infrastructures, Smart Grids, Interdependencies, Vulnerability Analysis, Uncertainty

\section{INTRODUCTION}

$\mathrm{T}$ HE life and development of modern Societies is supported by critical infrastructures (CIs), like the transport and communication networks or the electrical grid, which grant the necessary continuous production and distribution of goods and services [1].

A characteristic of these CIs is that they are highly interconnected and mutually dependent, i.e. interdependent, in complex ways, both physically and through information and communication technologies used for data acquisition and control, leading to the concept of "systems of systems" [2].

Manuscript received June 21, 2011.

E. Zio is Chair on Systems Science and the Energetic challenge, European Foundation for New Energy-EDF at Ecole Centrale Paris and Supelec, Paris, France. He is also with Energy Department, Politecnico di Milano, 20133 Milan, Italy (phone: 39-02-2399.6340; fax: 39-02-2399.6309; e-mail: enrico.zio@ecp.fr; enrico.zio@supelec.fr; enrico.zio@polimi.it).

G. Sansavini is with Energy Department, Politecnico di Milano, 20133 Milan, Italy (e-mail: giovanni.sansavini@polimi.it).
This brings the need of assessing and controlling the influences and limitations which interacting CIs impose on their operating conditions [3].

In particular, a focal subject of interest today is the evolution of the networks for electrical energy supply and their conception/renovation as "smart" grids [4]-[8] with distributed generation, as opposed to the centralized power generation structure of the existing electric power grids. One key aspect characterizing smart grids is the combination of the electric power grid with an information and communication network to "improve the observability and controllability of the distribution grid and thereby convert it from a static infrastructure to be operated as designed to a flexible, dynamic infrastructure operated proactively" [4].

The concepts and configurations of smart grids vary sensibly with respect to the implementation at the different levels of the electrical infrastructure, i.e. the transmission and distribution systems (the level of the individual costumer, and the related pricing issues, lies beyond the scope of the study presented in this paper) [5].

At the level of the transmission system, the efficiency and stability of power system operation could be improved substantially by phase angle measurements at many key locations and the use of Flexible AC Transmission System control devices (FACTS), combined with distributed and autonomous control strategies and high-speed communication.

The level of the distribution system is the one usually referred to when introducing concepts of smart grids. Unlike the transmission grids that are arranged as meshed networks, the distribution networks form tree-like, radial structures, characterized by a central distribution hub where power enters and is routed to the loads along the parallel branches of the distribution feeders. If a malfunctioning occurs, circuit breakers automatically disconnect the entire feeder. The use of multiple power injection points, and sensors and remote control switches that can isolate and cut off the problem would guarantee the continuous power supply to the other buses of the feeder [9]. Furthermore, at present stage the disconnection of the entire feeder is the only strategy to balance a load excess when generation is suddenly lost due to an emergency. Conversely, a combination of smart meters and advanced distribution automation would allow controlling individual loads along a distribution feeder so that critical services (such 
as police stations, hospitals, emergency services) can remain connected, while loads that provide less critical services can be dropped. Furthermore, the deployment of small-size diffuse generation would relieve stresses on transmission and distribution systems, and allow the disconnection of the distribution feeder from the main power system to run it as an isolated island, serving only a few critical loads in case of an emergency (this is not currently allowed for technical, legal, safety, and regulatory reasons).

Irrespective of the system level under consideration, the smart grid is an auto-balancing, self-monitoring power grid that has the ability to sense when a part of its system is overloaded and reroute power to reduce overload and prevent a potential outage situation. It enables real-time communication between users and utility, allowing optimal energy usage, and the introduction of renewable energy sources for minimizing the collectivity environmental footprint [4].

The use of renewable energy sources poses challenges to the design and the operations of the electric power grid. The availability of these sources is strictly dependent on the climatic conditions, i.e. cloud cover or wind speed and steadiness. Then, the power output of solar and wind electric generators is affected by uncertainty and intermittency [10][12]: paradoxically, due to its intrinsic stochastic nature, electric power from renewable sources could be abundantly available when it is not needed, while scarce in other cases of necessity. Moreover, electrical energy from renewable sources is typically produced in remote areas, e.g., where strong and steady winds are available or solar power plants can be conveniently located. This power has to be routed through the transmission network and delivered to distant locations where it is consumed. Yet, the existing power transmission infrastructure is not currently designed to carry intermittent electrical flows with possibly large peak flows, and in some cases it is inadequate to serve new remote power plants. As an example, a recent study [13] commissioned by the Italian Association of Energy Producers from Renewable Sources (APER) has estimated that between 2008 and 2009 the wind farms located in Southern Italy have lost $700 \mathrm{GWh}$ (25\% of the maximum producible energy) due to the limited currentcarrying capacity of the high-voltage transmission lines and the scarce power demand in the area near the plants, corresponding to an economic loss of 144 millions of Euros.

The deployment of small-size renewable power generation at the electric distribution level is also rapidly changing the nature of this infrastructure. Traditionally a passive recipient of a unidirectional flow from the transmission network, the distribution network should now support injections of active power from its users. In this perspective, both the transmission and the distribution networks require a real-time control approach with an increasing level of complexity due to the broad space distribution of the generators and to the high degree of variability that characterizes electrical power from renewable sources [6].
Electric transmission networks already have some instrumentation that allows control centers to automatically monitor power flows and open or close circuit breakers at substations as a result of human- and computer-generated control decisions [5]. This communication level is based on heterogeneous technologies and platforms which are poorly integrated [14]. On the contrary, distribution networks lack instruments for the information exchange and the system control.

Considering the present lack of standards, the communication network will have to evolve with the developing Smart Grid. Communication links will need to use all kinds of resources, varying from hard-wired links to fiber optics, power line communication [15], wireless [16], satellites and micro-wave links, in order to serve the large geographical territory covered by the smart grid [7]. All of these communication links constitute opportunities but also introduce vulnerabilities, due to functional failures and exposition to malevolent attacks aiming at causing systemwide instabilities and blackouts, especially if they are not protected in dedicated supports but can be accessed over the Internet.

The coupling between the electric power and communication networks in Smart Grids raises safety and security issues [17]. In the smart grids, the continuous delivery of electric power is supported by the communication infrastructure and, vice versa, the exchange of information relies on the energy input of the electric power grid. Faults originating in one of the two networks impact on the other interdependent one. For example, the interruption or even the delay of the information flow communicating a control action upon the electric power grid may result in power unbalance and system instability that could trigger a blackout [7], [18]. Vulnerability analysis must then be concerned with the interconnected, mutually dependent systems to properly account for the influences and limitations which the interacting electrical power grids and communication networks impose on the individual system operating conditions, for avoiding fault propagation by designing redundancies and alternative modes of operations, and by detecting and recognizing threats.

This paper looks into the vulnerabilities of the electric power grid and associated communication network, in the face of intermittent power generation and uncertain demand within a complex network framework of analysis of smart grids. Complex network approaches for the analysis of distributed systems [19]-[25] take a holistic perspective in which the overall system behavior emerges as the result of the complex interactions of its constituents. The two main objectives of the analysis are (i) to identify preliminary vulnerabilities by topology-driven and dynamic analysis, and (ii) to guide and focus further detailed analyses of critical areas based on physical codes (e.g. incorporating line impedances and Kirchhoff's laws to assess the power flows from the generators to the loads of an electric network). Purely-topological, complex network models of the electric power grid [19]-[25], 
which abstract the physical details of the provided service and characterize the topology of network systems, are here extended to capture essential operating features, i.e. the power is routed through the least resistant paths, the components are physically specialized in "generators" and "distributors", the lines are subjected to limits on their carrying-flow capacities and the power generation and consumption levels vary within a range of uncertain values.

In order to account for the interdependence effects due to the coupling [26], we adopt a framework in which the communication delays are integrated in the electric CI. The communication network is assumed to be made of dedicated control signal channels. The coupling between the two interdependent networks occurs at the components level. Each node of the electric network is coupled with a router that ensures communication with the other elements of the network. The time required to exchange data from the measurement location to a control center or data concentrator, and the time required ultimately to communicate these data to the control devices, is collectively denoted as communication delay or latency. As stated before, excessive communication delay may result in power unbalance and system instability that could trigger a blackout. The calculation method of the communication delay encompasses several sources, including routing delays which are modeled as a series of $M / M / 1$ queues, which symbolizes the cumulative time delay along the routing path from the measurement site to the control unit [27]. According to the shorthand notation developed for queuing systems, the three-part descriptor $M / M / 1$ denotes a one-server queuing system with exponentially-distributed inter-arrival and service times [28].

We assess the vulnerability of the two interdependent networks subject to the specific hazard defined by the request of increase of power generation due to enhanced power consumption of one load. To this aim, we introduce vulnerability measures that identify the most critical communication time delays, and assess the probability that a reduction in the functionality of the communication system will generate a faulty condition in the electric power grid.

The paper is organized as follows: the embraced interpretation of vulnerability is detailed in Section II; the modeling of the smart grid CI with interdependencies is presented in Section III; Section III.A describes the model of power flow in the electrical transmission network and Section III.B describes the model of information flow in the communication network; Section III.C details the simulation procedure devised for analyzing the vulnerabilities of the two coupled CIs. In Section IV, the proposed model is applied to two interdependent networks whose structures are based on the $380 \mathrm{kV}$ Italian power transmission network [29], [30], and Section IV.A discusses the numerical results. Conclusions are drawn in Section V.

\section{VULNERABILITY ANALYSIS OF CRITICAL INFRASTRUCTURES}

While the concept of risk is fairly mature and consensually agreed, the concept of vulnerability is still evolving and not yet established. In general terms, risk refers to a combination of the probability of occurrence of a specific event leading to loss, damage or injury and its extent. These quantities and their associated uncertainties are regarded as being numerically quantifiable.

The term vulnerability has been introduced as the concept of risk is somehow limited in portraying the hazard-centric perception of disasters. A hazard of low intensity could have severe consequences, while a hazard of high intensity could have negligible consequences: the level of vulnerability is making the difference [31]. The concept of vulnerability seen as a global system property focuses on three elements [32]: (i) degree of loss and damages due to the impact of a hazard; (ii) degree of exposure to the hazards, i.e., likelihood of being exposed to hazards of a certain degree and susceptibility of an element at the risk of suffering loss and damages; (iii) degree of resilience, i.e., the ability of a system to anticipate, cope with/absorb, resist and recover from the impact of a hazard or disaster.

Vulnerability is here interpreted as a flaw in the design, implementation or operation of an infrastructure system, or its elements, that renders it susceptible to destruction or incapacitation when exposed to a hazard or threat, or reduces its capacity to resume new stable conditions [1], [33]. The latter can be provided with a likelihood (frequency) while a measure for destruction or incapacitation needs specific elaborations. In this study, the vulnerability of the two interdependent networks is expressed in terms of frequency of load-shedding actions due to excessive communication time delay, with respect to several ranges of power increase request.

The goals of vulnerability analysis, and the associated modeling and simulation efforts, could be: $(i)$ given a system and the end state of interest, identify the set of events and event sequences that can cause damages and loss effects; (ii) identify the relevant set of "initiating events" and evaluate their cascading impact on a subset of elements, or the system as a whole; (iii) given a system and the end state of interest, identify the set of events or respective event sequences that would cause this effect; (iv) given the set of initiating events and observed outcomes, determine and elaborate on (inter)dependencies (within the system and among systems) and on coupling effects of different orders [1].

The achievement of these goals relies on the analysis of the system, its parts and their interactions within the system; the analysis must account for the environment which the system lives in and operates, and finally for the objectives the system is expected to achieve. The ultimate goal is to identify the vulnerabilities for managing and reducing them. 


\section{DYNAMIC MODEL OF INTERDEPENDENT NETWORKS AND SIMULATION PROCEDURE}

The electric power grid and the communication network are represented by two graphs $G_{l}\left(N_{l}, K_{l}\right)$ and $G_{2}\left(N_{2}, K_{2}\right)$ with $N_{l}$, $N_{2}$ nodes connected by $K_{1}, K_{2}$ links, respectively. In our case, it has been assumed (without loss of generality) that $N_{1}=N_{2}=N$ and $K_{I}=K_{2}=K$. The networks are specified by $N \times N$ adjacency (connection) matrices whose entries are 1 if there is an edge joining node $i$ to node $j$ or 0 , otherwise, and by connection length matrices whose entries are the physical length of the line connecting node $i$ to node $j$ if there is an edge joining node $i$ to node $j$ or 0 , otherwise.

In the electric power grid, the nodes correspond to buses. They are further specified into $N_{G}$ generators, i.e. the providers of the service, and $N_{D}$ distributors, i.e. the recipient of the service. The edges correspond to electrical connections.

In the communication network, the nodes correspond to routers and the edges to dedicated control signal channels that connect them.

The interdependencies within the $\mathrm{CI}$ are due to the presence of routers on each electrical bus. Routers send and receive control commands that try to match the generation profile with the power demand in the electrical network according to the increase of electrical load.

The system, then, does not rely on a centralized control center. Control decisions are locally taken at the router/bus level and communicated to the other interacting buses/routers [33]. During this process, the total time required to the system to balance generation and demand, i.e. the communication time delay, $T$, is monitored.

\section{A. The functional model of power flow in the electrical transmission network}

The functional model of the electric power grid abstracts the physical details while at the same time capturing its essential operating features. The elements of the network are characterized by loading values normalized in the interval $[0$, 1] that identify how close to the limit capacity they are operating. For generator and distributor nodes, $L^{i} \in[0,1]$ identifies the fraction of maximum power that generator $i$ is injecting in the network, or alternatively, the fraction of maximum power that distributor $i$ is absorbing from the network. The value $L^{i}=1$ identifies the maximum power that a generator $i$ can inject in the system, or alternatively, the maximum power that distributor $i$ has agreed to receive by contractual agreement. For lines, $L^{i j} \in[0,1]$ identifies the fraction of the maximum flow-carrying capacity that the line connecting node $i$ to node $j$ is serving. The value $L^{i j}=1$ indicates that the line connecting node $i$ to node $j$, hereafter also called line $i j$, is operating at its maximum flow carryingcapacity.

The loading scenario is identified when the $N$ values of $L^{i}$ and the $K$ values of $L^{i j}$ are known. To account for the variability that characterizes generation and consumption, the $L^{i}$ values are sampled from uniform probability distributions in the intervals $\left[L G^{\min }, L G^{\max }\right]$ for generators and $\left[L D^{\min }, L D^{\max }\right]$ for distributors.

We assume that the flow of electrical power from generator $i$ to distributor $j$ follows the least resistant path between $i$ and $j$. We further assume line resistance to be proportional to line length; hence the least resistant paths and the shortest paths coincide. We find the shortest paths that connect any generator $i$ to any distributor $j$, and rank in descending order the lines with respect to their participation to the shortest paths. The $L^{i j}$ values are then also sampled from uniform probability distributions. In particular, two probability distributions are assumed that describe two possible states $\left[L_{\text {low }}{ }^{\text {min }}, L_{\text {low }}{ }^{m a x}\right]$ for lines that are likely to accommodate low values of power, and $\left[L_{\text {high }}{ }^{\min }, L_{\text {high }}{ }^{{ }^{m a x}}\right]$ for lines that are likely to accommodate large values of power. To this aim, The $L^{i j}$ value is sampled from $\left[L_{h i g h}{ }^{\min }, L_{h i g h}{ }^{m a x}\right]$ if line $i j$ belongs to the first half of the rank, while it is sampled from $\left[L_{\text {low }}{ }^{m i n}, L_{\text {low }}{ }^{m a x}\right]$ if line ij belongs to the second half of the rank. This sampling procedure assigns on the average a higher power flow to those lines that are traversed by many generator-distributor shortest paths, i.e. that have a high betweenness centrality [36].

In the following, we assess how the CI copes with an increase in the electrical power demand. To this aim, starting from an initial loading scenario, we simulate a request of additional power, $W$, by distributor $i$. We assume that $W$ is described by a uniform probability distribution $\left[W^{\min }, W^{\max }\right]$. The overall load $L^{i}+W$ may also possibly exceed the maximum contractual power that $i$ has agreed to receive from the system, i.e. 1 .

\section{B. The model of information flow in the communication network}

In [27], Stahlhut et al. assess how the communication delay for measurements and control signals in a power system impacts the control system response. In the following, we embrace their calculation of the communication delay, and assume that the data transmitted are in the form of packets. The packets are a formatted block of information and are typically arranged in three sections: the header, the payload, and the trailer. The header has the following information: packet length, origin and destination address, packet type, and packet number (if a sequence of packets is being sent). The payload carries the data taken from the measurement. The trailer is at the end of the packet and carries information which permits the receiving device to identify the end of the packet. The time delay calculation encompasses several different delays that occur in communication systems. These delays typically are:

- serial delays: the delay of having bits being sent one after another;

- "between packet" serial delays: the time after a packet is sent to when the next packet is sent;

- propagation delays: the time required to transmit data over a particular communication medium;

- routing delays: the time required for data to be sent through a router, and resent to another location. 
The total signal time delay may be represented as

$$
\begin{aligned}
& T=T_{s}+T_{b}+T_{p}+T_{r} \\
& T_{s}=P_{s} / D_{r} \\
& T_{P}=l / v
\end{aligned}
$$

where $T_{s}$ is the serial delay, $T_{b}$ is the between packet delay, $T_{p}$ is the propagation delay, $T_{r}$ is the routing delay, $P_{s}$ is the size of the packet (bits/packet), $D_{r}$ is the data rate of the network, $l$ is the length of the communication medium, and $v$ is the velocity at which the data are sent through the communication medium (e.g., 0.6 to $c$, where $c$ is the speed of light).

Routers are a major part of the communication infrastructure in a communication network. There are a number of methods by which routing delay can be approximated [37]-[41]. A simple and fast calculation method of routing delay can be used to approximate the delay at a router: this calculation method is based on a series of $M / M / 1$ queues [28], in which a path from the measurement to the control center is traced, and all of the routing delays are added up to represent the total routing delay for the measurement. For this calculation,

$T_{r}=\sum_{i=1}^{N} T_{r}^{(i)}$

where $T_{r}$ is the total routing delay, $T_{r}^{(i)}$ is the routing delay for a single router at location $i$, and $N$ is the total number of routers. The $M / M / 1$ queue is used to approximate the value of $T_{r}^{(i)}$ for each router $i$, and it is modeled as a one-server queuing system with exponentially-distributed inter-arrival and service times [28]. The $M / M / 1$ queue has several associated performance measures such as: the average number of customers in line, the average number of customers in the system, the time waiting in line, and the time required to pass through the system [39], [42]. In this context, the customers are the sensory messages in the communication network. A noteworthy performance measure is the total waiting time (system time), and this encompasses both the amount of time waiting in line (waiting time), as well as the amount of time being served (service time). The total waiting time is quantified as

$$
\frac{\lambda}{\lambda \cdot(\mu-\lambda)}
$$

where $\lambda$ is the rate at which objects come in the system (e.g., packets/s) and $\mu$ is the rate at which the objects are being served (e.g., packets/s). The routing time $T_{r}^{(i)}$ for each router $i$ can be estimated from (5) and used in (4) to calculate the total routing delay
$T_{r}=\sum_{i=1}^{N} \frac{\lambda^{(i)}}{\lambda^{(i)} \cdot\left(\mu^{(i)}-\lambda^{(i)}\right)}$

We assume that information from router $i$ to router $j$ flows along the shortest path connecting $i$ and $j$. Therefore, the total routing time, $T_{r}$, from router $i$ to router $j$ is evaluated as the sum of the routing delays of the encountered routers along the shortest path connecting $i$ and $j$.

The maximum time delay that the smart grid can tolerate depends on the system task to be executed and ranges from tenths of milliseconds up to tenths of seconds [6], [7]. For example, the standard specifies that for an unintentional island in which the distributed generation energizes a portion of the distribution network, the control system shall detect the island and cease to energize the portion within $2 \mathrm{~s}$ of the formation of an island. Moreover, latencies in the tens of ms allow rapid detection of faults and are the accepted fault detection times [7]. On the contrary, voltage and active power regulation actions should be accomplished within 10s and some minutes, respectively [6].

\section{Simulation procedure}

To test the vulnerability of the CI with respect to the stability upon a power increase request, $W$, we proceed in successive stages as follows:

1. All $N$ components of the electric power grid are working under independent uniformly random initial loads. In particular, $L^{i} \in\left[L G^{\min }, L G^{\max }\right]$ for generator $i=1, \ldots N_{G}, L^{i} \in$ $\left[L D^{\min }, L D^{\max }\right]$ for distributor $i=1+N_{G}, \ldots N_{D}+N_{G}$, and $L^{i j}$ $\in\left[L_{\text {high }}{ }^{\text {min }}, L_{\text {high }}{ }^{\text {max }}\right]$, or $L^{i j} \in\left[L_{\text {low }}{ }^{\text {min }}, L_{\text {low }}{ }^{\text {max }}\right]$, if line $i j$ belongs to the first or second half of the betweenness centrality rank (Section III.A), respectively, $i, j=1, \ldots, N$. These values identify the loading scenario.

2. A distributor node $i$ is chosen randomly and its power demand is increased from $L^{i}$ to $L^{i}+W$, where $W$ is sampled from the uniform probability distribution $\left[W^{\text {min }}, W^{\max }\right]$.

3 . The $N_{G}$ generators that are connected to $i$ are sorted by ascending distance from $i$, i.e. ascending resistance. The closest generator $j=1$ is selected. Communication delay $T$ is set to zero.

4. Distributor $i$ queries generator $j$ for additional power $W$. Generator $j$ answers providing the value of the power available to $i$, i.e. $S G(j)=\min \left(W, 1-L^{j}\right)$, where $1-L^{j}$ is the maximum additional power that $j$ can provide. Communication time delay, $T$, is updated.

5. Check whether the shortest path between $i$ and $j$ can accommodate the additional power $S G(j)$. To this aim, every line $y z$ on the shortest path between $i$ and $j$ is tested for infringement of the current-carrying capacity limit in the new configuration, i.e. $L^{y x}+S G(j) \leq 1$. If at least one limit is exceeded the additional power is updated to the maximum power value that satisfies all the line constraints, i.e. 
$S G(j)=\min _{y z}\left(1-L^{y z}\right)$, for every line $y z$ on the shortest path

between distributor $i$ and generator $j, S G(j)=0$ in case that a line $y z$ is already operating at its maximum flow-carrying capacity. The residual power that distributor $i$ still requires, $U(i)$, and the power that generator $j$ could not supply, $U G(j)$, are evaluated as $U(i)=U G(j)=W-\sum_{s=1}^{j} S G(s)$. They are recorded for the vulnerability evaluation. Communication time delay, $T$, is updated.

6. The generator index $j$ is incremented by 1 , and the procedure is returned to step 4 until $U(i)=0$, in case of a successful system state, or $j=N_{G}$ and $U(i)>0$, in case the power request is not satisfied. The final communication time delay, $T$, is recorded.

The value of $U(i)$ at the end of the simulation is the fraction of the additional load $W$ that has to be shed in order to balance the power generation and the power consumption. Therefore, we assume that load can be continuously shed.

Furthermore, it can be noticed that the power that generator $j$ could not supply, $U G(j)$ can be greater than 0 for two different causes: either $j$ is operating at its maximum capacity, or there is no path for the power produced by $j$ for reaching the distributor $i$ where it is needed. This feature of the algorithm is consistent with the behavior of smart grids that include intermittent and remotely-generated electricity from renewable energy sources, as discussed in Section I.

To incorporate the effects of load and generation uncertainties, the above algorithm is embedded in a Monte Carlo simulation framework, in which a large number of additional load requests, e.g., 100000 in this study, is simulated for the same range of $\left[L G^{\min }, L G^{\max }\right],\left[L D^{\min }, L D^{\max }\right]$, $\left[L_{\text {high }}{ }^{\text {min }}, L_{\text {high }}{ }^{m a x}\right],\left[L_{\text {low }}{ }^{\text {min }}, L_{\text {low }}{ }^{\text {max }}\right]$ and $\left[W^{\text {min }}, W^{\text {max }}\right]$, in order to obtain statistically significant results for various realizations of the same average loading condition.

During the simulations, relevant statistics are recorded to evaluate different vulnerability indexes, i.e. the distribution of the communication time delay, $T$, in case of a successful (no load-shedding is required to balance consumption and generation) or unsuccessful (load-shedding is required to balance consumption and generation) final system state.
The effects of the interdependencies between the electric power grid and the communication network are evaluated with respect to the communication time delay, $T$, that elapses from the instant in which the request for power increase is done, until the request is fulfilled. As seen in Section III.B, the maximum time delay requirements, $T^{M A X}$, for the smart grid range from tenths of milliseconds up to tenths of seconds depending on the system task to be executed. In this respect, various communication network configurations differing for the value of the data rate, $D_{r}$, can be tested to quantify the probability that a malfunction in the communication system, i.e. a communication delay, will generate a faulty condition in the electric power grid. The compliance with $T^{M A X}$, i.e. $T<$ $T^{M A X}$, is evaluated at the end of the simulations.

\section{CASE STUDY}

The approach to evaluate the vulnerability of the smart grid CI with interdependencies between power and communication networks introduced in Section III, is exemplified with reference to the topological network of the $380 \mathrm{kV}$ Italian power transmission network (Fig. 1), focusing only on its structure with no further reference on the electrical properties, coupled with a dedicated communication network. For simplicity, but with no loss of generality, we assume that the two networks have the same topologies. The developed methodology can be applied to interdependent networks with different topologies. The $380 \mathrm{kV}$ Italian power transmission network is a branch of a high voltage level transmission, which can be modeled as a network of $N=127$ nodes $\left(N_{G}=30\right.$ generator and $N_{D}=97$ distributor nodes) connected by $K=171$ links [29], [30], defined by its $N \times N$ adjacency (connection) matrix $a_{i j}$ and connection length matrix $l_{i j}$. We assume that the communication network consists of dedicated control signal channels, which follow the same layout of the overhead electrical lines. Therefore, both the electric power grid and the communication network are described by the same $a_{i j}$ and $l_{i j}$ matrices (without loss of generality): the topology and the geographical location of the $380 \mathrm{kV}$ Italian power transmission network components serve as reference. 


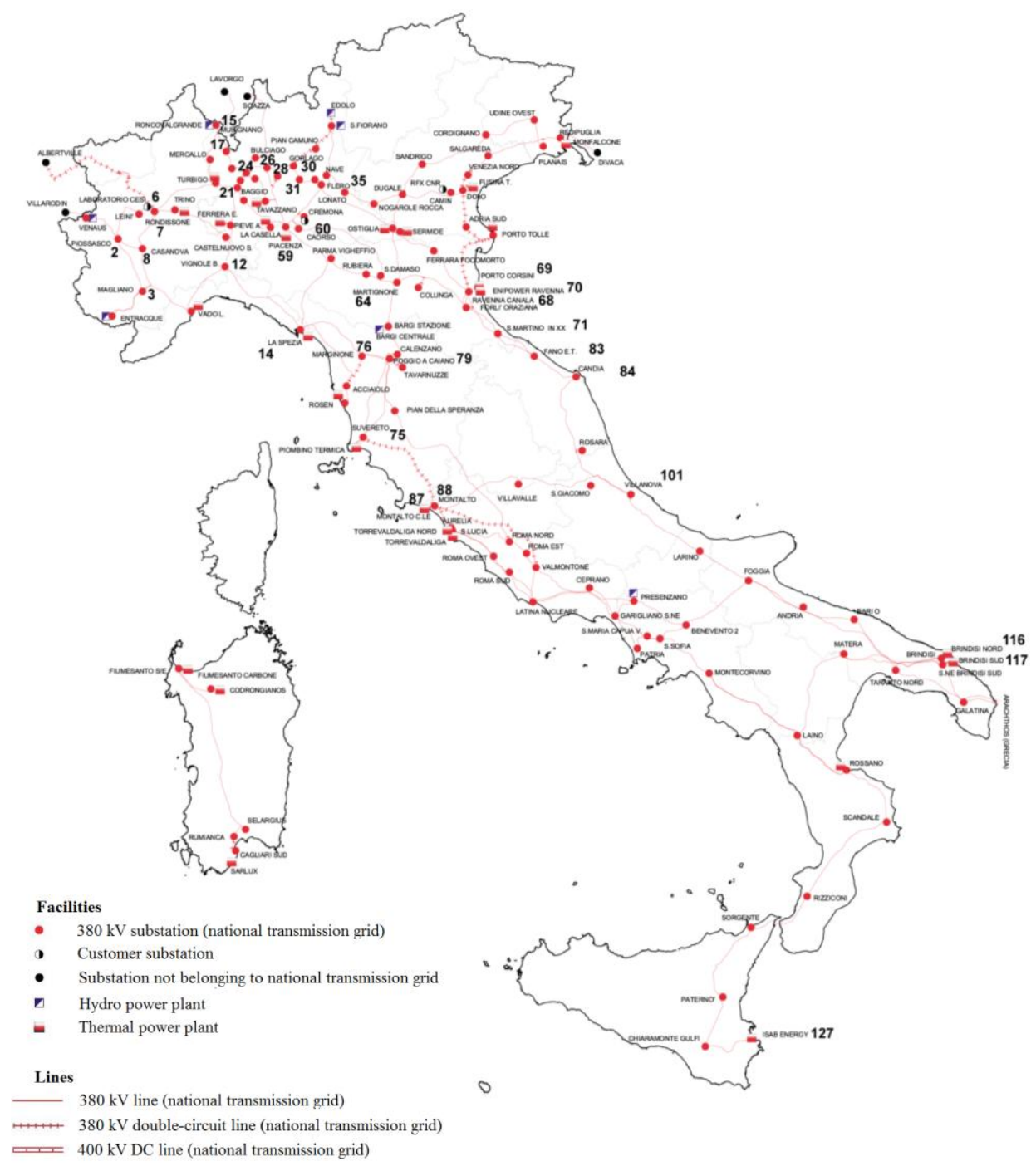

Fig. 1. The $380 \mathrm{kV}$ Italian power transmission network [29], [30].

We test the vulnerability of the two interdependent networks with respect to the stability upon a power increase request, $W$, for eight increasing ranges of $\left[W^{\min }, W^{\max }\right]$ reported in Table II, while the other system parameters are constant and specified in Table I. We assume that the electric power grid operates with an average $20 \%$ safety margin for all the components except for the highly loaded lines which operate with an average $15 \%$ safety margin. The data for the communication network have been taken from [27] and assume a dedicated communication network.

TABLE I

SPECIFICATIONS OF THE ELECTRIC POWER GRID AND OF THE COMMUNICATION NETWORK USED IN THE SIMULATIONS

\begin{tabular}{ll|ll}
\hline \hline$L G^{\min }$ & 0.7 & Data rate of the network, $D_{\mathrm{r}}$ & $5 \mathrm{Mbps}$ \\
$L G^{\max }$ & 0.9 & Between packet delay, $T_{b}$ & 0 \\
$L D^{\min }$ & 0.7 & Packet size, $P_{s}$ & $2 \mathrm{~kb}$ \\
$L D^{\max }$ & 0.9 & Data velocity, $v$ & $0.6 \mathrm{c}$ \\
$L_{\text {high }}{ }^{\text {min }}$ & 0.8 & Measurement rate, $\lambda$ & 50 packets/s
\end{tabular}

$$
\begin{array}{ll|ll}
L_{\text {high }}{ }^{\max } & 0.9 & \text { Router serving rate, } \mu & 50 \mathrm{Mbps} \\
\text { Llow }_{\text {min }} & 0.7 & \\
\text { Llow }_{\text {max }} & 0.9 & & \\
\hline
\end{array}
$$

Then, we test the vulnerability of the two interdependent networks with respect to the stability upon a power increase request, $W \in\left[W^{\text {min }}, W^{\text {max }}\right]$, for thirteen values of data rate of the network, $D_{r}$, in Table III which identify different dedicated network configurations for the communication CI. We aim at quantifying the probability that a malfunction in the communication system, i.e. a communication delay, will generate a faulty condition in the electric power grid.

\section{A. Numerical results}

Table II reports the frequency of unsatisfied power request, i.e. load-shedding intervention, with respect to the eight sampling intervals $\left[W^{\min }, W^{\max }\right]$. As $W^{\max }$ increases, a greater amount of additional load $W$ is requested on the average and 
the generators closest to the increasing load cannot provide the total required amount. Thus, distant generators are queried, but power cannot always find a path to reach the increasing load due to growing likelihood of line limit infringement along the path, and the system has little chances to fully satisfy $W$.

\section{TABLE II}

FREQUENCY OF UNSATISFIED POWER REQUEST, I.E. LOAD-SHEDDING INTERVENTION, AND THE ASSOCIATED STANDARD ERROR VS. THE WIDTHS OF THE EIGHT UNIFORM SAMPLING INTERVALS FOR THE ADDITIONAL POWER, $W$. THE NUMBER OF SIGNIFICANT DIGITS THAT WE REPORT THROUGHOUT THE PAPER IS EVALUATED BY YONEDA'S RULE [43].

\begin{tabular}{c|c|c}
\hline \hline $\begin{array}{c}\text { Sampling interval for the } \\
\text { additional power request, } W\end{array}$ & $\begin{array}{c}\text { Frequency of } \\
\text { unsatisfied request }\end{array}$ & Standard error \\
\hline$[0.10,0.15]$ & $0.6670 \%$ & $0.1252 \%$ \\
{$[0.10,0.20]$} & $1.1950 \%$ & $0.1263 \%$ \\
{$[0.10,0.25]$} & $2.6000 \%$ & $0.1717 \%$ \\
{$[0.10,0.30]$} & $6.565 \%$ & $0.384 \%$ \\
{$[0.10,0.35]$} & $11.788 \%$ & $0.316 \%$ \\
{$[0.10,0.40]$} & $17.587 \%$ & $0.554 \%$ \\
{$[0.10,0.45]$} & $23.083 \%$ & $0.425 \%$ \\
{$[0.10,0.50]$} & $28.835 \%$ & $0.553 \%$ \\
\hline \hline
\end{tabular}

[Fig. 2-7 in the previous manuscript were removed in order to comply with the 12 page limit for IEEE Transactions on Systems, Man and Cybernetics, Part A-Systems and Humans regular papers. Table 3-6 in the previous manuscript were removed in order to comply with the 12 page limit for IEEE Transactions on Systems, Man and Cybernetics, Part A Systems and Humans regular papers. The comments to the removed results were also removed.]

The frequency of unsatisfied power request in Table II characterizes the self-limitations that the electric power grid imposes on its own operations due to its structural configuration, the limits on flow-carrying capacities of lines, the intermittent nature of power generation from renewable energy sources and the uncertainties in the load demand profile. We now devote our attention to the temporal aspects that characterize the power balance procedure in the CI. In this respect, Fig. 2 shows the probability distribution function of the communication time delay, $\operatorname{PDF}(T)$, when the request to increase load is not satisfied by generators, i.e. $U>0$, for the eight sampling intervals $\left[W^{\min }, W^{\max }\right]$ in Table II. Fig. 2 is relative to situations in which the additional load request $W$ could not be met by generators, and the communication signal spans the entire extension of the network to query for additional generation capability. This appears from the larger $T$ values in the $\operatorname{PDF}(T)$ plot, which are the communication time delays needed to route the power to the increasing load bus from remote regions of the network.

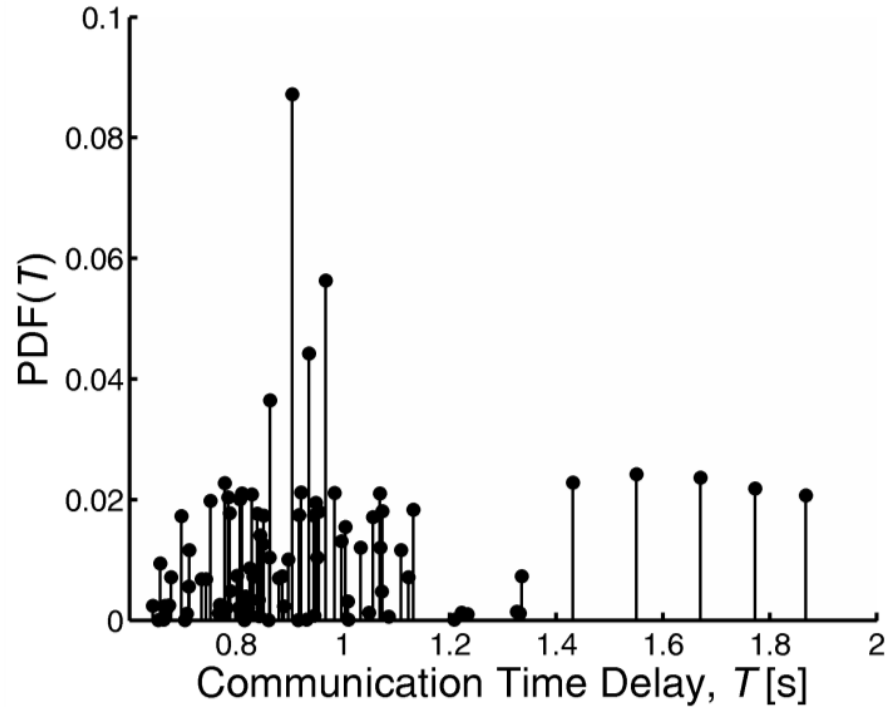

Fig. 2. Probability distribution function of the communication time delay, $\operatorname{PDF}(T)$, when the request to increase load is not satisfied by generators, i.e. $U$ $>0$, for the eight sampling intervals $\left[W^{\min }, W^{\max }\right]$ in Table II.

The differences in the communication time delay, $T$, distribution for normal operations and load shedding appear in Fig. 3, where the $\operatorname{CDF}(T)$ 's are plotted for these two situations and encompass the eight sampling intervals $\left[W^{\min }, W^{\max }\right]$ in Table II. We expect that $\operatorname{CDF}(T)$ for normal operations (solid curve in Fig. 3) is shifted to smaller $T$ values than the one relative to load-shedding interventions (dashed curve in Fig. 3 ). When the extra power request is satisfied, $U=0$, only a fraction of the total number of generators is queried for additional power. On the contrary, when the extra power request is not satisfied, $U>0$, the communication signal spans the entire generation set looking for additional generation capability that is not found.

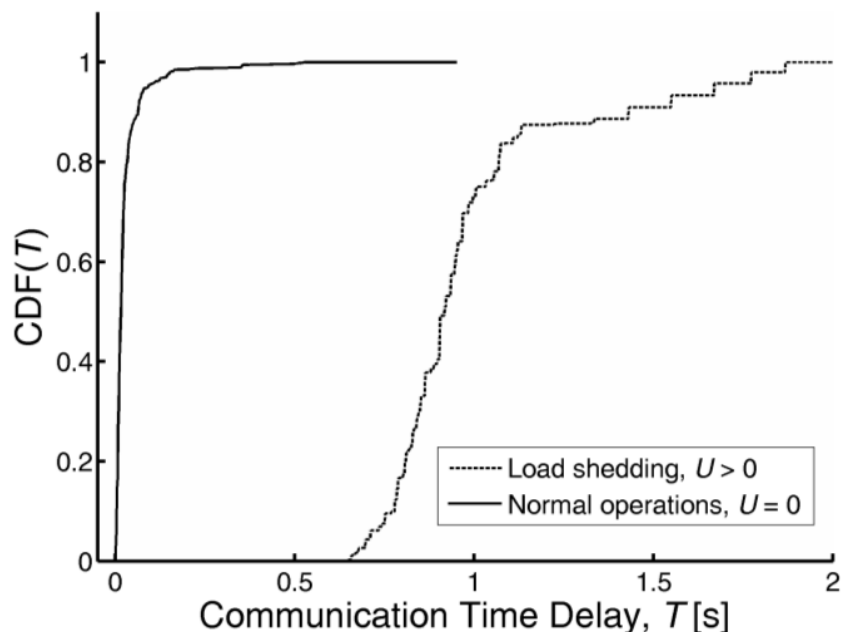

Fig. 3. Cumulative distribution function of the communication time delay, $T$, when the request to increase load is not satisfied, i.e. $U>0$ (dashed line), and when the request to increase load is fully satisfied by generators, i.e. $U=0$ (solid line), for the eight sampling intervals $\left[W^{\min }, W^{\max }\right]$ in Table II.

The communication time delay, $T$, is distributed within the intervals $T \in[62.6,951] \mathrm{ms}$ and $T \in[643,2000] \mathrm{ms}$, in case of normal operations and of load-shedding actions, respectively. 
Given the maximum admissible communication time delay, $T^{M A X}$, Fig. 3 allows identifying the frequency of load-shedding due to the excess of communication latency, i.e. $T>T^{M A X}$, even for those loading scenarios that would eventually result in $U=0$ otherwise (solid line in Fig. 3). As detailed in Section III.B, $T^{M A X}$ depends on the system task to be executed and ranges from tenths of milliseconds up to tenths of seconds. In this context, $T^{M A X}$ can be thought of as the maximum time delay before instabilities arise in the electric power grid due to the imbalance between generation and consumption. If the value $T^{M A X}$ equal to $100 \mathrm{~ms}$ is assumed, we evaluate $\operatorname{CDF}(T=$ $\left.T^{M A X}=100 \mathrm{~ms}\right)=96.77 \% \pm 0.06 \%$, and we discover that due to latency constraints the frequency of faulty conditions is $3.23 \% \pm 0.06 \%$ even for those scenarios that we classified as normal operations because the additional power request is fully satisfied, i.e. $U=0$ (solid line in Fig. 3). If a narrower latency constraint is assumed, e.g., $T^{M A X}=50 \mathrm{~ms}$, we evaluate $\operatorname{CDF}\left(T=T^{M A X}=50 \mathrm{~ms}\right)=87.85 \% \pm 0.10 \%$, and conclude that the frequency of faulty conditions is $12.2 \% \pm 0.10 \%$ even for those scenarios that we classified as normal operations because the additional power request is fully satisfied, i.e. $U=0$.

The frequency of faulty conditions due to the excess of communication time delay, $T$, quantifies the limitations that the communication system imposes on the operations of the interdependent electric system. It can be used to measure the strength of the interdependency or the degree of the coupling between these two systems within the CI.

After having found an index that quantifies the extent to which the delay in the communication network impacts on the operations of the electric power grid, we investigate how the frequency of faulty conditions due to the excess of communication time delay, $T$, varies with respect to different configurations of the communication network. We test several networks characterized by decreasing values of the data rate, $D_{r}$ (Table III first column), that is defined as the number of bits that are conveyed or processed per unit of time. Since higher investments correspond to higher $D_{r}$, this kind of analysis allows identifying the optimal communication network that respects the constraint relative to the frequency of faulty conditions due to the excess of communication time delay, $T$. Alternatively, we can evaluate the extent to which a malfunction that reduces the speed of communication, $D_{r}$, will impact on the power grid due to the interdependencies between the two systems.

Fig. 4 shows the cumulative distribution functions of the communication time delay, $T$, for decreasing values of the data rate of the communication network, $D_{r}$, reported in Table III first column, and $W \in[0.10,0.25]$. These results correspond to scenarios of the electric power grid for which a final balance between generation and consumption could be found, i.e. $U=$ 0 . Fig. 4 allows evaluating the extent to which $D_{r}$ values influence the degree of coupling between the two CIs. Smaller $D_{r}$ values produce $\operatorname{CDF}(T)$ that are shifted to larger $T$ values. If we assume a maximum admissible communication time delay, $T^{M A X}$, from Fig. 4 we identify the frequency of load-shedding due to the excess of communication latency, i.e. $T>T^{M A X}$, even for those loading scenarios that would eventually result in $U$ equal to 0 otherwise. As an example, if $T^{M A X}$ is equal to 100 $\mathrm{ms}$, the scenarios that are found to the right of the vertical dash-dotted line in Fig. 4 will require load-shedding activity.

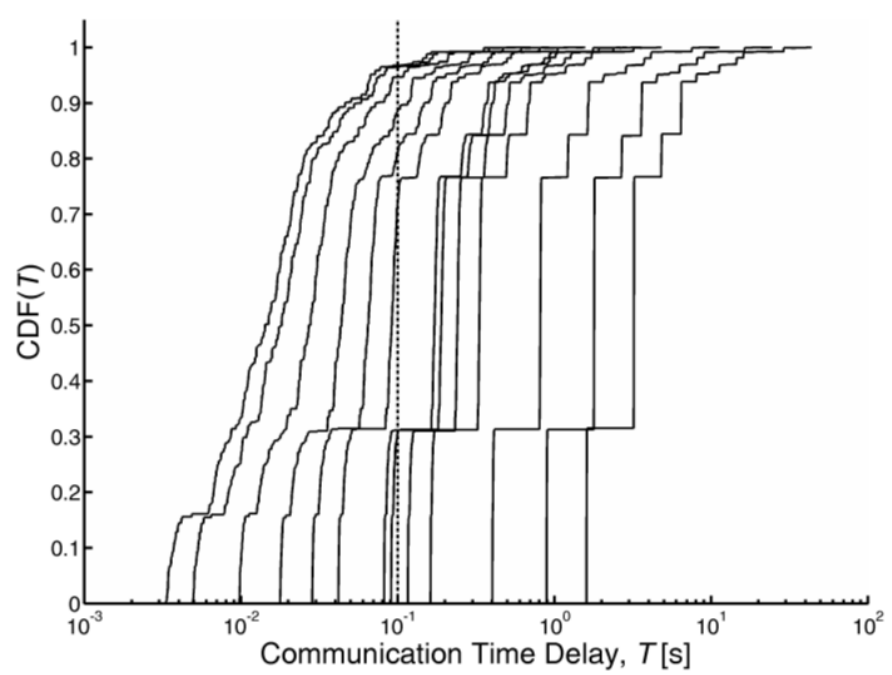

Fig. 4. Cumulative distribution functions of the communication time delay, $T$, for decreasing values of the data rate of the communication network, $D_{r}$, reported in Table III and $W \in[0.10,0.25]$. Smaller $D_{r}$ values yield distributions shifted towards larger $T$ values. The vertical dash-dotted line indicates the maximum allowable communication time delay, $T^{M A X}=100 \mathrm{~ms}$.

We quantify the effects of the interdependency between the two networks by two complementary indexes that provide safety margins with respect to the coupled operations of the two CIs. The $95^{\text {th }}$ percentile of the distribution of $T$ quantifies the minimum admissible time delay, $T^{M A X}$, that limits to $5 \%$ the frequency of load-shedding due to excessive latency. From Table III second column and Fig. 5, we see how the $95^{\text {th }}$ percentile varies with $D_{r}$.

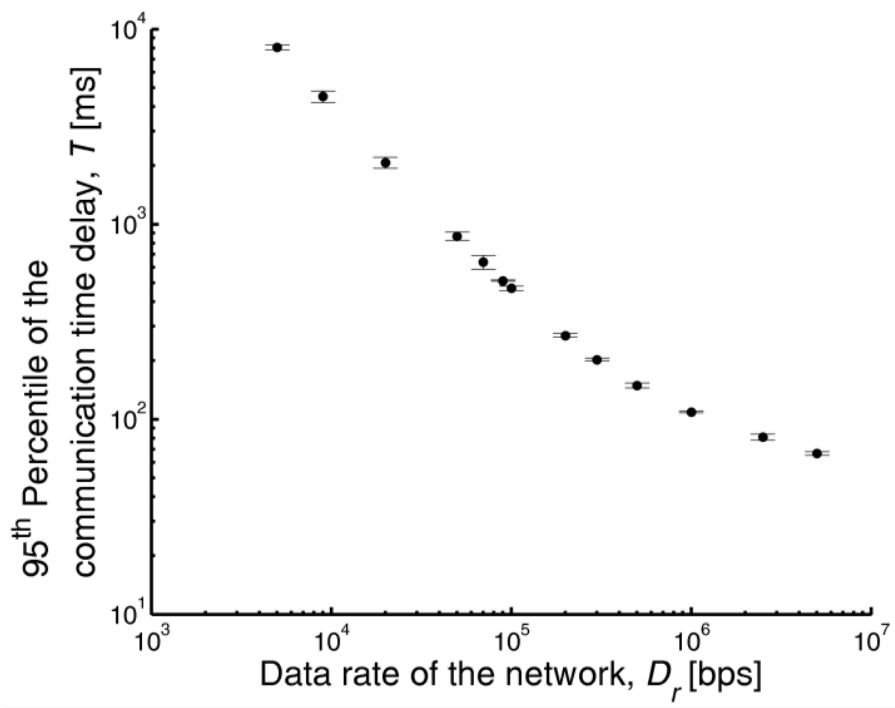

Fig. 5. $95^{\text {th }}$ percentile of the communication time delay, $T$, vs. the values of the data rate of the communication network, $D_{r}$, and $W \in[0.10,0.25]$.

For small $D_{r}$, e.g., $D_{r}=2 \cdot 10^{4} \mathrm{bps}$, the system must tolerate 
a minimum latency $T^{M A X}$ greater than or equal to $2.069 \mathrm{~s} \pm$ $0.140 \mathrm{~s}$, in order to limit to $5 \%$ the frequency of load-shedding due to excessive latency. In other words, power increase operations that must be safely carried out within a time smaller than $2.069 \mathrm{~s} \pm 0.140 \mathrm{~s}$ should not be attempted, if one wants to comply with the safety margin of 5\% load-shedding frequency. Conversely, if $D_{r}$ is equal to $5 \cdot 10^{6} \mathrm{bps}$, the system can admit a minimum latency as small as $T^{M A X}$ equal to or greater than $0.6678 \mathrm{~s} \pm 0.0149 \mathrm{~s}$, and the frequency of load-shedding due to excessive latency would still be limited to $5 \%$.

TABLE III

$95^{\mathrm{TH}}$ PERCENTILE OF THE COMMUNICATION TIME DELAY, $T$, (SECOND COLUMN) AND PROBABILITY THAT $T \leq T^{M A X}$ (THIRD COLUMN) WITH STANDARD ERRORS, FOR DECREASING VALUES OF THE DATA RATE OF THE COMMUNICATION NETWORK, $D_{R}$, (FIRST COLUMN) AND $W \in[0.10,0.25]$.

\begin{tabular}{ccc}
\hline \hline $\begin{array}{c}\text { Data rate of the } \\
\text { network, } D_{r}[\mathrm{bps}]\end{array}$ & $\begin{array}{c}95^{\text {th }} \text { Percentile of the } \\
\text { communication time } \\
\text { delay, } T[\mathrm{~ms}]\end{array}$ & $\begin{array}{c}\mathrm{P}\left(T \leq T^{\text {MAX }}\right)=\mathrm{CDF}\left(T=T^{\text {MAX }}\right. \\
=100 \mathrm{~ms})\end{array}$ \\
\hline $5 \cdot 10^{6}$ & $66.789 \pm 1.489$ & $96.77 \% \pm 0.06 \%$ \\
$2.5 \cdot 10^{6}$ & $80.94 \pm 2.95$ & $96.47 \% \pm 0.06 \%$ \\
$1 \cdot 10^{6}$ & $108.986 \pm 1.029$ & $94.69 \% \pm 0.07 \%$ \\
$5 \cdot 10^{5}$ & $148.97 \pm 4.31$ & $88.89 \% \pm 0.10 \%$ \\
$3 \cdot 10^{5}$ & $202.22 \pm 3.25$ & $81.14 \% \pm 0.13 \%$ \\
$2 \cdot 10^{5}$ & $268.91 \pm 6.27$ & $72.48 \% \pm 0.14 \%$ \\
$1 \cdot 10^{5}$ & $468.9 \pm 12.5$ & $31.18 \% \pm 0.15 \%$ \\
$9 \cdot 10^{4}$ & $513.33 \pm 3.31$ & $30.99 \% \pm 0.15 \%$ \\
$7 \cdot 10^{4}$ & $640 \pm 53$ & $0 \%$ \\
$5 \cdot 10^{4}$ & $869 \pm 41$ & $0 \%$ \\
$2 \cdot 10^{4}$ & $2069 \pm 140$ & $0 \%$ \\
$9 \cdot 10^{3}$ & $4513 \pm 300$ & $0 \%$ \\
$5 \cdot 10^{3}$ & $8069 \pm 200$ & $0 \%$ \\
\hline \hline
\end{tabular}

Because the admissible latency $T^{M A X}$ is a constraint in the system, a somewhat more intuitive safety margin is provided with respect to $\mathrm{P}\left(T \leq T^{M A X}\right)=\operatorname{CDF}\left(T=T^{M A X}\right)$, i.e. the probability of final safe conditions (no load-shedding is required), given the admissible latency $T^{M A X}$. In Table III third column and Fig. 6, $\mathrm{P}\left(T \leq T^{M A X}=100 \mathrm{~ms}\right)=\operatorname{CDF}\left(T=T^{M A X}=\right.$ $100 \mathrm{~ms}$ ) values are presented as functions of $D_{r}$, given $W \in$ $[0.10,0.25]$ and $T^{M A X}=100 \mathrm{~ms}$. From Table III third column, we see that when $D_{r}=1 \cdot 10^{5} \mathrm{bps}$, the probability of final safe conditions is $31.18 \% \pm 0.15 \%$ and, consequently, the frequency of load-shedding due to excessive latency is $68.82 \%$ $\pm 0.15 \%$.

Fig. 6 displays how the probability of final safe conditions decreases as $D_{r}$ progressively decreases from $D_{r}=1 \cdot 10^{6} \mathrm{bps}$ to $D_{r}=5 \cdot 10^{3}$ bps. $\mathrm{P}\left(T \leq T^{M A X}=100 \mathrm{~ms}\right)$ exhibits a

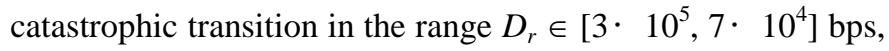
as $D_{r}$ values decrease below $3 \cdot 10^{5}$ bps. No final safe condition is realized below $D_{r}=7 \cdot 10^{4}$ bps, i.e. every additional power request, $W$, involves some load-shedding actions due to excessive latency in the communication, $T$ > $T^{M A X}$.

The information in Fig. 6 can be used during the design phase of the CI with the aim of identifying the communication specifications that comply with the required safety standards, given $T^{M A X}$, i.e. the smallest admissible time delay, and $\left[W^{\min }\right.$,
$\left.W^{m a x}\right]$, i.e. the electric load profile. In our example, a communication network with $D_{r} \geq 1.26 \cdot 10^{6}$ bps has to be selected to comply with the $95 \%$ safety margin, i.e. $5 \%$ probability of load-shedding, when $T^{M A X}=100 \mathrm{~ms}$ and $W \in$ $[0.10,0.25]$. Furthermore, the information in Fig. 6 can be used during operations to quantify the extent to which a possible loss of quality in the communication, i.e. a $D_{r}$ reduction, impacts on the electric power grid. In our example, a $D_{r}$ reduction from $D_{r}=5 \cdot 10^{6} \mathrm{bps}$ (normal operations) to $D_{r}$ $=1.94 \cdot 10^{5}$ bps (anomalous operations), entails a reduction of the safety margin from $96.77 \% \pm 0.06 \%$ to approximately $70 \%$, i.e. the probability of load-shedding increases from $3.23 \% \pm 0.06 \%$ to approximately $30 \%$. Yet, most important is the fact that an additional small $D_{r}$ reduction to $D_{r}=7 \cdot 10^{4}$ bps entails the complete disappearance of the safety margin. Therefore, the knowledge of the relation in Fig. 6 is vital for operating the $\mathrm{CI}$ with interdependencies in a region of the system parameters that is far from the catastrophic phase transition point.

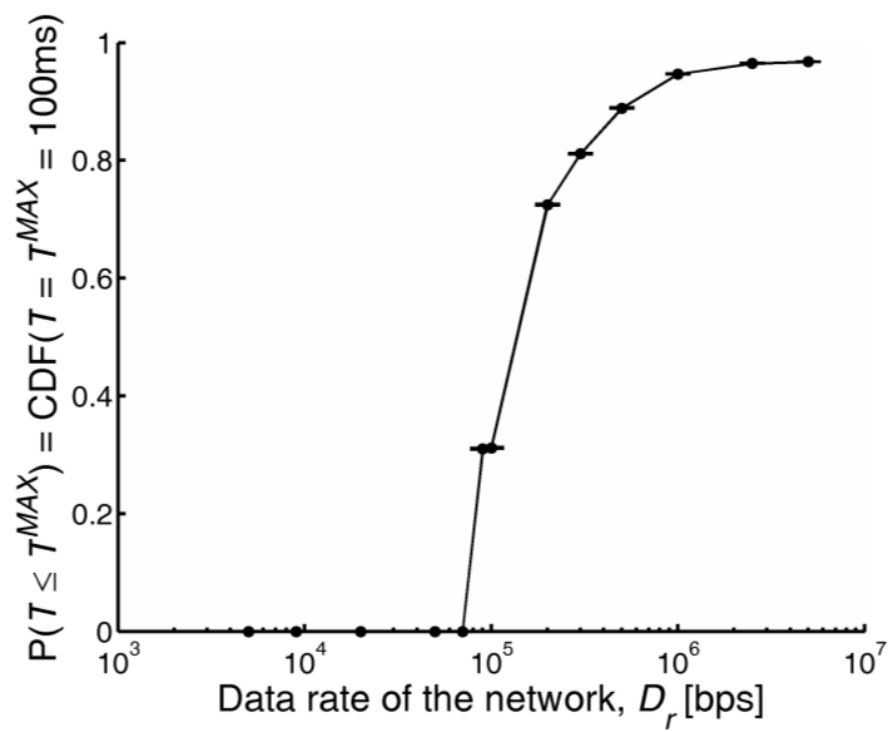

Fig. 6. Probability of final safe conditions, i.e. $\mathrm{P}\left(T \leq T^{M A X}\right)=\operatorname{CDF}(T=$ $T^{M A X}=100 \mathrm{~ms}$ ), given the admissible latency $T^{M A X}$, vs. data rate of the communication network, $D_{r}$, and $W \in[0.10,0.25]$.

\section{CONCLUSIONS}

In this paper, we have looked into the vulnerabilities that arise when complex networks are coupled, with reference to smart grids modeled as a combination of the electric power grid and the communication network. We have adopted a modeling framework in which the communication delays are integrated in the electric CI. The complex network approach proposed extends the purely-topological models of the electric power grid to capture essential operating features, i.e. the power is routed through the least resistant paths, the components are physically specialized in "generators" and "distributors", and the lines are subjected to limits on their carrying-flow capacities; moreover, it includes the further 
complexity that arises from the variability of power generation from renewable energy sources and intermittent load consumption. However, given the somewhat abstract level of the modeling supporting complex network analysis, the insights gained with respect to the vulnerable areas in the system (first findings) may not be clear-cut, and major hidden vulnerabilities may still be expected. Then, to accurately model the physical behavior of the interconnected systems, computational frameworks that propagate the flows of power and information based on physical laws have to be embraced, and more detailed information about the system and its operating environment may be needed. For large, real-scale systems, this requires the development of adequate computational methodologies through clustering computing, co-operative simulation, or similar architectures [44]-[48].

We have quantified the limitations that both the electric power grid and the communication network impose on the operations of the CI with interdependencies, when a load bus demands additional power from the generators. In the application to the $380 \mathrm{kV}$ Italian power transmission network, with the assumptions made and the numerical data used, we have observed that a factual indicator for quantifying the coupling strength between the two integrated systems is the frequency of load-shedding actions due to excessive communication time delay. By means of this indicator, we have evaluated the extent to which a loss of quality in the communication impacts on the electric power grid, and we have selected appropriate communication specifications, i.e. the data rate of the network, that comply with the required safety requirements in the electric power grid.

Finally, we have detected a catastrophic phase transition point in the frequency of faulty conditions with respect to the data rate of the communication network. The CI with interdependencies safely operate only in a region of the system parameters that are far from this catastrophic transition point. To this aim, the introduction of adequate safety margins with respect to the data rate of the communication network is suggested.

In summary, the main contributions to the knowledge generated for the network general case are: (i) the extension of the purely-topological complex network modeling framework by inclusion of relevant physical aspects of the system; (ii) the use of the frequency of load-shedding actions due to excessive communication time delay as a quantitative indicator for quantifying the vulnerability of the smart grid; (iii) the detection of a catastrophic phase transition with respect to the data rate of the communication network in the smart grid.

\section{[APPENDIX]}

[Appendix was removed because it was related to the results of Section IV.A which were also removed.]

\section{ACKNOWLEDGMENT}

The authors are very thankful to the four anonymous reviewers for helping in improving the paper through their observations and suggestions.

\section{REFERENCES}

[1] W. Kröger and E. Zio, Vulnerable Systems. London, UK: Springer London, 2011.

[2] S. M. Rinaldi, "Modeling and simulating critical infrastructures and their interdependencies," in Proc. Thirty-Seventh Annu. Hawaii Int. Conf. Syst. Sci., Jan. 5-8, 2004, Waikoloa, Big Island, Hawaii, Computer Society Press, 2004.

[3] R. Zimmerman, "Social implications of infrastructure network interactions," J. Urban Technol., vol. 8, no. 3, pp. 97-119, Dec. 2001.

[4] J. Heckel, "Smart Substation and Feeder Automation for a SMART Distribution Grid," in Proc. CIRED 2009 - 20 th Int. Conf. and Exhibition on Electr. Distrib. - Part 1, Jun. 8-11, 2009, London, UK: The Institution of Engineering and Technology, 2009.

[5] M. Granger Morgan, J. Apt, L. B. Lave, M. D. Ilic, M. Sirbu, and J. M. Peha, The many meanings of "Smart Grid". A Briefing Note from the Department of Engineering and Public Policy, Carnegie Mellon University, Jul. 2009 - url: http://repository.cmu.edu/epp/22/

[6] Smart Grid Laboratory - Energylab, Smart Grid. Le reti elettriche di domani. Milano, Italy: Gruppo Italia Energia (Gieedizioni), 2011 (in Italian).

[7] V. K. Sood, D. Fischer, J. M. Eklund, and T. Brown, "Developing a communication infrastructure for the Smart Grid," in Proc. IEEE Electr. Power Energy Conf. (EPEC), Oct. 22 - 23, Montréal, Canada, IEEE, 2009.

[8] M. D. Ilić, L. Xie, U. A. Khan, and J. M. F. Moura, "Modeling of Future Cyber-Physical Energy Systems for Distributed Sensing and Control," IEEE Trans. Syst., Man, Cybern. A, Syst., Humans, vol. 40, no. 4, pp. 825-838, Jul. 2010.

[9] A. Ababnah and B. Natarajan, "Optimal Control-Based Strategy for Sensor Deployment," IEEE Trans. Syst., Man, Cybern. A, Syst., Humans, vol. 41, no. 1, pp. 97-104, Jan. 2011.

[10] S. Conti and S. Raiti, "Probabilistic load flow using Monte Carlo techniques for distribution networks with photovoltaic generators," Sol. Energy, vol. 81, no. 12, pp. 1473-1481, Dec. 2007.

[11] M. H. Albadi and E. F. El-Saadany, "Overview of wind power intermittency impacts on power systems," Electr. Power Syst. Res., vol. 80, no. 6, pp. 627-632, Jun. 2010.

[12] Y. Wang and L. Li, "Effects of Uncertainty in Both Component Reliability and Load Demand on Multistate System Reliability," IEEE Trans. Syst., Man, Cybern. A, Syst., Humans, to be published.

[13] F. Zanellini, Rete e vento - Lo sviluppo della rete elettrica italiana per la connessione e l'integrazione della fonte eolica. Milano, Italy: Centro Studi APER - REEF Onlus, May 2011 (in Italian).

[14] The Advisory Council of 'SmartGrids' - European Technology Platform for the Electricity Network of the Future, "Strategic Deployment Document for Europe's Electricity Networks of the Future," Apr. 2010 - url: http://www.smartgrids.eu/documents/SmartGrids_SDD_FINAL_ APRIL2010.pdf

[15] S. Galli, A. Scaglione, and Z. Wang, "For the Grid and Through the Grid: The Role of Power Line Communications in the Smart Grid," Proc. IEEE, vol. 99, no. 6, pp. 998-1027, Jun. 2011.

[16] S. W. Lai, G. G. Messier, H. Zareipour, and C. H. Wai, "Wireless network performance for residential demand-side participation," in Proc. IEEE PES Conf. Innovative Smart Grid Technol. Eur. (ISGT Europe), October 11-13, 2010, Gothenburg, Sweden, IEEE Power and Energy Society, 2010.

[17] C.-W. Ten, G. Manimaran, and C.-C. Liu, "Cybersecurity for Critical Infrastructures: Attack and Defense Modeling," IEEE Trans. Syst., Man, Cybern. A, Syst., Humans, vol. 40, no. 4, pp. 853-865, Jul. 2010.

[18] C. Peng, Y.-C. Tian, and D. Yue, "Output Feedback Control of Discrete-Time Systems in Networked Environments," IEEE Trans. Syst., Man, Cybern. A, Syst., Humans, vol. 41, no. 1, pp. 185-190, Jan. 2011.

[19] I. Dobson, B. A. Carreras, and D. E. Newman, "A loading-dependent model of probabilistic cascading failure," Probab. Eng. Inf. Sci., vol. 19, no. 1, pp. 15-32, 2005.

[20] H. Hines and S. Blumsack, "A Centrality Measure for Electrical Networks," in Proc. $41^{\text {st }}$ Annu. Hawaii Int. Conf. Syst. Sci., Jan. 7-10, 2008, Waikoloa, Big Island, Hawaii, Computer Society Press, 2008. 
[21] P. Holme, "Edge overload breakdown in evolving networks," Phys. Rev. $E$, vol. 66, no. 3, pp. 036119, 2002.

[22] P. Kinney, P. Crucitti, R. Albert, and V. Latora, "Modeling cascading failures in the North American power grid," Eur. Phys. J. B, vol. 46, no. 1, pp. 101-107, 2005.

[23] A. E. Motter and Y.-C. Lai, "Cascade-based attacks on complex networks," Phys. Rev. E, vol. 66, no. 6, pp. 065102(R), 2002.

[24] J. Wu, M. Barahona, Y.-J. Tan, and H.-Z. Deng, "Spectral Measure of Structural Robustness in Complex Networks," IEEE Trans. Syst., Man, Cybern. A, Syst., Humans, vol. 41, no. 6, pp. 1244-1252, Nov. 2011.

[25] W.-C. Yeh, "An Improved Method for Multistate Flow Network Reliability With Unreliable Nodes and a Budget Constraint Based on Path Set," IEEE Trans. Syst., Man, Cybern. A, Syst., Humans, vol. 41, no. 2, pp. 350-355, Mar. 2011.

[26] M. G. Kallitsis, G. Michailidis, and M. Devetsikiotis, "A Framework for Optimizing Measurement-Based Power Distribution under Communication Network Constraints," in Proc. First IEEE Int. Conf. Smart Grid Commun. (SmartGridComm), Gaithersburg, Maryland, USA, 4-6 Oct. 2010, Institute of Electrical and Electronic Engineers, 2010.

[27] J. W. Stahlhut, T. J. Browne, G. T. Heydt, and V. Vittal, "Latency Viewed as a Stochastic Process and its Impact on Wide Area Power System Control Signals," IEEE Trans. Power Syst., vol. 23, no. 1, pp. 84-91, 2008.

[28] L. Kleinrock, Queuing g systems. New York, USA: John Wiley \& Sons, Inc., 1975.

[29] Terna S.p.A. - Rete Elettrica Nazionale, Dati statistici sull'energia elettrica in Italia, 2002. (in Italian) - url: http://www.terna.it/LinkClick.aspx?fileticket=PUvAU57MlBY\%3d\&ta bid=41 8\&mid $=2501$

[30] V. Rosato, S. Bologna, and F. Tiriticco, "Topological properties of highvoltage electrical transmission networks," Electr. Power Syst. Res., vol. 77, no. 2, pp. 99-105, 2007.

[31] G. F. White, Natural hazards: local, national and global. New York, NY: Oxford University Press, 1974, p. 288

[32] S. Bouchon, "The vulnerability of interdependent critical infrastructures systems: epistemological and conceptual state-of-the-art," EU Commission, Joint Research Centre, Ispra, Italy, EU Report, 2006.

[33] A. M. Cramer, S. D. Sudhoff, and E. L. Zivi, "Metric OptimizationBased Design of Systems Subject to Hostile Disruptions," IEEE Trans. Syst., Man, Cybern. A, Syst., Humans, vol. 41, no. 5, pp. 989-1000, Sep. 2011.

[34] J. Johansson and H. Hassel, "An approach for modelling interdependent infrastructures in the context of vulnerability analysis," Rel. Eng. Syst. Safety, vol. 95, no. 12, pp. 1335-1344, Dec. 2010.

[35] E. Camponogara, D. Jia, B. H. Krogh, and S. Talukdar, "Distributed model predictive control," IEEE Control Syst. Mag., vol. 22, no. 1, pp. 44-52, 2002.

[36] A. Tizghadam and A. Leon-Garcia, "Betweenness Centrality and Resistance Distance in Communication Networks," IEEE Netw., vol. 24, no. 6, pp. 10-16, 2010.

[37] J. Irvine and D. Harle, Data Communications and Networks. West Sussex, UK: Wiley, 2001.

[38] R. M. Metcalfe, Packet Communication. San Jose, CA: Peer-to-peer Communications, 1996.

[39] J. Daigle, Queuing Theory with Applications to Packet Telecommunication. New York, USA: Springer Science, 2005.

[40] K. Hopkinson, X. Wang, R. Giovanini, J. Thorp, K. Birman, and D. Coury, "EPOCHS: A platform for agent-based electric power and communication simulation built from commercial off-the-shelf components," IEEE Trans. Power Syst., vol. 21, no. 2, pp.548-558, 2006.

[41] F.-L. Lian, J. R. Moyne, and D. M. Tilbury, "Performance evaluation of control networks: Ethernet, ControlNet, and DeviceNet," IEEE Control Syst. Mag., vol. 21, no. 1, pp. 66-83, 2001.

[42] D. Gross and C. M. Harris, Fundamentals of Queuing Theory. New York, NY: Wiley, 1998.

[43] W. T. Song and B. W. Schmeiser, "Omitting Meaningless Digits in Point Estimates: The Probability Guarantee of Leading-Digit Rules," Oper. Res., vol. 57, no. 1, pp. 109-111, 2009.
[44] Advances in Computers - Parallel, Distributed, and Pervasive Computing, A. R. Hurson and M. V. Zelkovits eds., vol. 63, San Diego, Ca, USA: ELSEVIER Inc., 2005.

[45] M. Verhoef, P. Visser J. Hooman, and J. Broenink, "Co-simulation of Distributed Embedded Real-Time Control Systems," in Proc. $6^{\text {th }}$ Int. Conf. Integr. Formal Methods, IFM 2007, Oxford, UK, July 2-5, 2007, J. Davies and J. Gibbons (Eds.), Berlin, Germany: Springer-Verlag, 2007.

[46] C. Jiang, X. Xu, and J. Wan, "Grid Computing Based Large Scale Distributed Cooperative Virtual Environment Simulation," in Proc. $12^{\text {th }}$ Int. Conf. Comput. Supported Cooperative Work in Design, Xi'an, Cina, 16-18 April 2008, Piscataway, NJ, USA: IEEE Press, 2008.

[47] T. Tomura, S. Kanai, and T. Kishinami, "A Cooperative Simulation Mechanism of Distributed Control Systems based on Object-Oriented Design Patterns," in Proc. $6^{\text {th }}$ IEEE Int. Symposium on Object-Oriented Real-Time Distrib. Comput., Hakodate, Hokkaido, Japan, 14-16 May 2003, Piscataway, NJ, USA: IEEE Press, 2003.

[48] P. A.F. Ferreira, E. F. Esteves, R. J.F. Rossetti, and E. C. Oliveira, "A Cooperative Simulation Framework for Traffic and Transportation Engineering," in Proc. $5^{\text {th }}$ Int. Conf. Cooperative Design, Visual., Eng., Calvià, Mallorca, Spain, September 21-25, 2008, Berlin, Germany: Springer-Verlag, 2008.

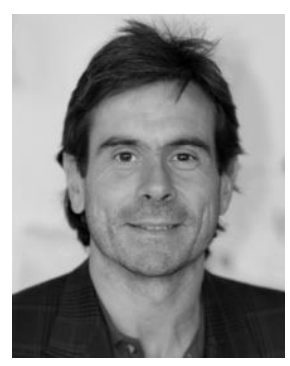

Enrico Zio (BS in nuclear engng., Politecnico di Milano, 1991; MSc in mechanical engng., UCLA, 1995; $\mathrm{PhD}$, in nuclear engng., Politecnico di Milano, 1995; PhD, in nuclear engng., MIT, 1998) is Director of the Chair in Complex Systems and the Energetic Challenge of the European Foundation for New Energy of Electricite' de France (EDF) at Ecole Centrale Paris and Supelec, full professor, President and Rector's delegate of the Alumni Association and past-Director of the Graduate School at Politecnico di Milano, adjunct professor at University of Stavanger. He is the Chairman of the European Safety and Reliability Association ESRA, member of the scientific committee of the Accidental Risks Department of the French National Institute for Industrial Environment and Risks, member of the Korean Nuclear society and China Prognostics and Health Management society, and past-Chairman of the Italian Chapter of the IEEE Reliability Society. He is serving as Associate Editor of IEEE Transactions on Reliability and as editorial board member in various international scientific journals, among which Reliability Engineering and System Safety, Journal of Risk and Reliability, International Journal of Performability Engineering, Environment, Systems and Engineering, International Journal of Computational Intelligence Systems. He has functioned as Scientific Chairman of three International Conferences and as Associate General Chairman of two others. His research focuses on the characterization and modeling of the failure/repair/maintenance behavior of components, complex systems and critical infrastructures for the study of their reliability, availability, maintainability, prognostics, safety, vulnerability and security, mostly using a computational approach based on advanced Monte Carlo simulation methods, soft computing techniques and optimization heuristics. He is author or co-author of five international books and more than 170 papers on international journals.

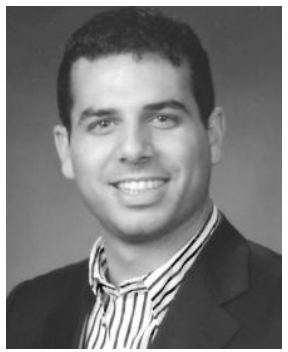

Giovanni Sansavini (B.S. in energy engineering, Politecnico di Milano, 2003; M.Sc. in nuclear engineering, Politecnico di Milano, 2005; Ph.D., in nuclear engineering, Politecnico di Milano, 2010; Ph.D., in engineering mechanics, Virginia Tech, 2010) is a Postdoctoral Research Assistant at the Department of Energy, Politecnico di Milano. His research interest is the analysis of the reliability, safety, and security of complex systems under stationary and dynamic operation. 